\title{
IV. THE VISCOSITY OF BLOOD.
}

\author{
BY J. W. TREVAN. \\ From the Physiological Laboratory, St Bartholomew's Hospital. \\ (Received November 24,191\%.) \\ I. INTRODUCTORY.
}

Several attempts have been made to correlate the viscosity of blood with the number of corpuscles present [Denning and Watson, 1906; Josué and Maturier, 1916]. All observers agree that the viscosity rises with increases in the number of corpuscles present and that the effect becomes progressively greater with each such increase above a certain critical value. It has also been noticed that blood from different animals shows different viscosities even when the plasma viscosity and the number of corpuscles are the same in each. These observations suggested that the factor contributed by the corpuscles depended on their total volume rather than on their number [McClendon, 1916, p. 180]. Ferrai [quoted by Denning and Watson, 1906] also, who showed that the addition of carbon dioxide to blood caused a rise in viscosity, attributed the rise to a simultaneous increase in the volume of the corpuscles. Thus a qualitative relationship has been established between the total volume of the corpuscles, the viscosity of plasma and the viscosity of whole blood; this paper is an endeavour to make clear the quantitative relationships.

\section{METhods.}

Home-made capillary viscosimeters were used of the pattern illustrated in Fig. $1 a$. They are blown from tubing of $0.8 \mathrm{~mm}$. diameter for the large size, using $2 \mathrm{cc}$., and $0.2 \mathrm{~mm}$. diameter for the small form, holding $1 \mathrm{cc}$. In the large model the bulb $C$ holds rather less than 1 cc. The other dimensions are in proportion. The funnel $D$ is made from a half-inch test-tube drawn out and fused on, and holds about five cubic centimetres. It is necessary to have it of that size to prevent overflow of bubbles, which may form when the blood is blown out of $C$ as described later. Two cc. of blood mixed with a little hirudin is placed in $D$ and allowed to run into $C$. The instrument is then placed upright in a thermostat at $40^{\circ}$. for ten minutes. The bulb $C$ is 
then emptied into $D$ by blowing slowly down the rubber tube $E$ until the whole of the blood is in $D$. The corpuscles are mixed with the plasma by blowing a gentle stream of air through or by stirring with a coiled wire ${ }^{1}$. The bubbles which form are destroyed by touching them with a hot needle and the time of flow from $B$ to $A$ noted with a stopwatch, the blood in $D$ being continually stirred with a coiled wire.

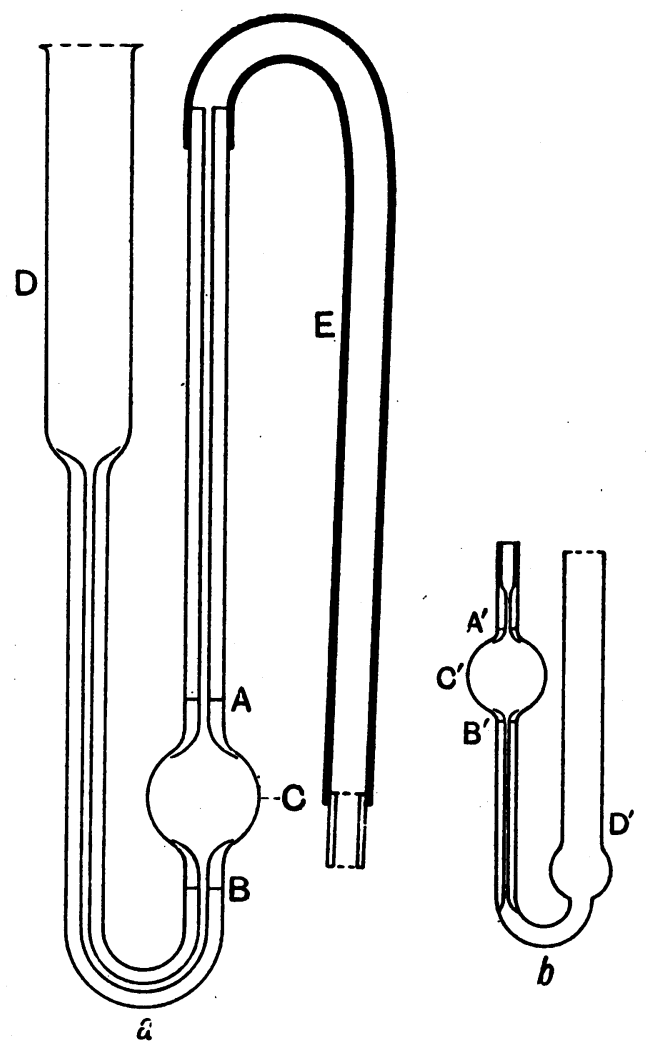

Fig. 1.

III. Causes of Error.

(a) Conditions setting up turbulent flow. The transition between the expanded portions and the capillary itself must be a gradual one and especially must not be constricted. One instrument constructed led to much waste of time because of this latter failing.

1 Blowing air through is of course inadmissible when the effect of amall changes in the $\mathrm{CO}_{2}$ content of the blood is in question but all the experiments to be described have been conducted with the blood fully oxygenated, and the changes in viscosity dealt with are very much greater (by as much as $1000 \%$ ) than the small changes produced by alterations in the viscosity of the blood. 
Not much emphasis is laid on this point in the usual textbook descriptions of viscosity measurements. The magnitude of the error is shown by the following figures (Table I) showing the relation of the time of flow of $7 \%$ gum acacia solution in $2 \%$ sodium chloride (used as having a viscosity similar to that of normal blood) to the time of flow of water at different temperatures and the ratio which gives the "apparent" viscosity.

\section{Table I.}

A is a viscosimeter with a constriction, B one without.

\begin{tabular}{|c|c|c|c|c|c|c|}
\hline \multirow{3}{*}{$\begin{array}{c}\text { Temp. } \\
40^{\circ} \\
16 \cdot 5^{\circ} \\
11 \cdot 5^{\circ}\end{array}$} & \multicolumn{2}{|c|}{$\begin{array}{c}\text { A } \\
\text { Time of flow }\end{array}$} & \multicolumn{4}{|c|}{$\begin{array}{c}\text { B } \\
\text { Time of flow }\end{array}$} \\
\hline & $\begin{array}{r}\text { Water } \\
12.5\end{array}$ & Gum & Ratio & Water & Gum & Ratio \\
\hline & $\begin{array}{c}12 \cdot 5 \\
19 \\
-\end{array}$ & $\begin{array}{c}43 \cdot 5 \\
82 \\
-\end{array}$ & $\begin{array}{l}\mathbf{3} \cdot 4 \\
\mathbf{4} \cdot \mathbf{3} \\
-\end{array}$ & $\frac{10}{18 \cdot 0}$ & $\frac{43}{7 \cdot 9}$ & $\frac{4 \cdot 3}{4 \cdot 45}$ \\
\hline
\end{tabular}

On blowing out the constriction the results were concordant. The error was caused by. the formation of eddies at the constriction. Turbulent flow was set up and the amount of energy absorbed by the eddies increases with the velocity of flow and is greater the less viscous the fluid [Osborne Reynolds, 1883]. Consequently the time of flow is increased more for the less viscous fluid and especially at the higher temperatures where the velocities of flow are higher. The "apparent" viscosity of very much more viscous fluids such as the gum solution used in the above experiment is therefore less than the real. Viscosimeters of large bore (greater than $0.5 \mathrm{~mm}$.) should always be calibrated by measuring not only the time of flow of water but also that of gum solutions of known viscosities (as measured in a very fine bore viscosimeter) including the whole range of viscosities to be measured. The use of very fine bore viscosimeters for the measurement of the viscosity of blood introduces chances of large errors due to settling of the corpuscles.

(b) The sedimentation of the corpuscles. Settling of corpuscles during the course of the transpiration has not attracted the attention it deserves. The rate varies enormously in different species and considerably in different individuals of the same species. The differences depend on no single physical factor. One of the most striking features is the slow settling which sometimes takes place in saline. The size of the error can be seen in the following table.

TABLE II.

Time of flow of sheep 8 corpuscles in saline; haematocrit volume $30 \%$.

Sedimentation prevented

Time of flow

$33 \cdot 4, \quad 33 \cdot 2, \quad 33 \cdot 8$
Sedimentation not prevented Time of flow

35.8, $35 \cdot 8$ (error ca. $10 \%$ ) 
This suspension settled rather rapidly. The error with a larger proportion of cotpuscles (longer times of flow) is a much greater one, for the greater the number of corpuscles present the greater the rate of increase of the viscosity with further increase in concentration (see p. 68). Without stirring I have found it impossible to get concordant results with the higher concentrations of corpuscles used. Nor does the fact that the later portions of the suspension to pass through the capillary tube contain a smaller number of corpuscles than the earlier compensate for this effect: for the transference of a volume of corpuscles from a dilute suspension to a stronger one produces a greater alteration of the viscosity of the strong suspension than of the dilute one (see p. 68). This may be crudely illustrated by the fact that it takes longer to empty a room full of people if everybody jostles around the doorway than if each waits his turn.

The form of viscosimeter usually adopted, with the measuring bulb on the pressure side of the viscosimeter $\left(A^{\prime} B^{\prime}\right.$, Fig. $\left.1 b\right)$ prevents any stirring during the course of the experiments. This led I think to the differences in "apparent" viscosity which Denning found between large tubes and small. I have been unable to obtain these differences working with tubes of $0.8 \mathrm{~mm}$. and $0.2 \mathrm{~mm}$. diameter. The narrower tubes in their experiments produced a long time of outflow which gave greater opportunities for settling and the higher the concentration reached in any experiment the greater the deviation from the real time of flow, the percentage error increasing during the experiment.

(c) Formation of beads of fluid. On making measurements with the instrument (Fig. $1 a$ ) care must be taken that beads of fluid do not collect in the capillary above $A$ (Fig. $1 a$ ). These form from the fluid, which wets the sides of the capillary, if $C$ is emptied too rapidly into $D$. A similar bead may form at $B$ and persisting as a bubble on the top of the inflowing blood, may pass into the capillary above $A$ as a bead. These beads of fluid require very large amounts of energy to drive them along capillary tubes, especially if they contain air bubbles (cf. air embolism in the coronary arteries); and when they reach the open end of the tube a bubble forms and breaks several times. Both these processes increase the time of flow very considerably. In the type of viscosimeter, Fig. $1 b$, these beads forming there at $A^{\prime}$ are even more troublesome.

(d) Temperature variations produce alterations in the viscosity of blood and the alterations are not parallel with that of water; all comparisons were therefore made at the same temperature, viz. $40^{\circ}$. 
(e) Use of anticoagulants. In these experiments hirudin was used. A few fine flakes were placed in the bottom of a short test-tube into which $5 \mathrm{cc}$. of blood were run. The hirudin was without appreciable influence on the viscosity, as was shown by collecting fowl's blood directly from an artery into a clean glass vessel, measuring its viscosity, adding hirudin and measuring the viscosity again. Defibrination is inadmissible because of its effect on the plasma. Oxalate, citrate and fluoride in the solid state are also inadmissible because of the alteration in the volume of the corpuscles by osmotic changes. The following table gives the alterations produced by the addition of $0.08 \%$ $\mathrm{NaCl}$ (corresponding to $0.1 \%$ potassium oxalate and $0.35 \%$ sodium citrate) in the corpuscular volume [Hamburger, 1905] and the consequent alteration in viscosity calculated on the assumption that the plasma viscosity remained constant (see formula established later, p. 68).

\section{TABLE III.}

\begin{tabular}{|c|c|c|c|c|}
\hline \multicolumn{2}{|c|}{ Corpuscular volume } & \multicolumn{2}{|c|}{ Viscosity as a multiple of water } & \multirow[b]{2}{*}{$\%$ error } \\
\hline before adding salt & after adding salt $0.08 \%$ & before adding salt & after adding salt & \\
\hline $\begin{array}{l}33.3 \% \\
48.6 \% \\
68.3 \%\end{array}$ & $\begin{array}{l}36.7 \% \\
63.1 \% \\
64.1 \%\end{array}$ & $\begin{array}{l}5.0 \\
6 \cdot 6 \\
9 \cdot 06\end{array}$ & $\begin{array}{l}4 \cdot 61 \\
5 \cdot 92 \\
7 \cdot 85\end{array}$ & $\begin{array}{r}9.8 \% \\
10 \cdot 1 \% \\
12.4 \%\end{array}$ \\
\hline
\end{tabular}

On this point see also Burton-Opitz [1911]. In employment of potassium oxalate other complications arise. At certain concentrations potassium oxalate produces large increases in the viscosity of the plasma, large enough sometimes to mask the effect mentioned above. Sodium citrate does not appear to produce this effect. The addition of isotonic solutions of sodium citrate to blood produces no osmotic changes, but causes difficulty by diminishing the viscosity of the plasma to an unknown extent. If the volume of the corpuscles is known, the true viscosity can be calculated by one of the formulae given later, but the method is obviously very cumbrous.

(f) Haemolysis by bacteria and saponin produces very large rises in viscosity. Centrifuged corpuscles on being allowed to stand in the air, become converted into a tarry mass which will not pour at all. Saponin also produces haemolysis with rise of viscosity (Table IV).

\section{Table IV.}

Time of flow

64.4 secs. before adding saponin, $0.020 \mathrm{~g}$.

80.0 " immediately after

276.0 " five minutes later 
The rise is proportional to the amount of saponin added as is shown in Table V.

\section{TABLE V.}

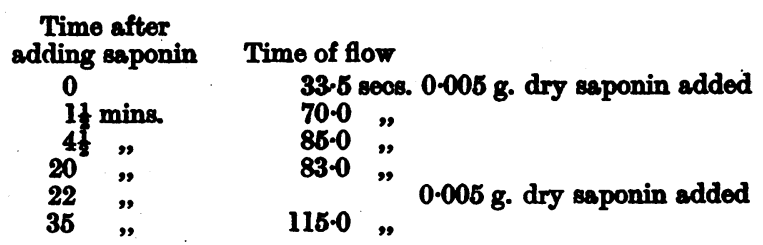

The viscosity is said by Burton-Opitz to be diminished by laking produced by freezing and thawing; but the condition which is most likely to be met with in the body is more akin to that of haemolysis by saponin.

\section{iV. Measurement of the Corpuscular Volume.}

The measurement of the volume of the corpuscles was carried out by centrifuging to a constant volume in a graduated tube, such as a haematocrit tube, with an ordinary electrical centrifuge working at 2500-3500 revolutions per minute. The graduated tube was closed by putting a piece of paper on the end and dipping into melted paraffin. A much firmer joint was thus made, than the leather pad and screw clip generally supplied. The time for complete sedimentation varies but is usually less than twentyfive minutes. The figures given in the following pages were obtained by centrifuging for fifty minutes. These measurements were necessarily made at room temperature, but by immersing the haematocrit tube in water at $40^{\circ}$ it was found that the proportion between the volume of the corpuscles and of the plasma remained the same.

\section{The Contribution of the Plasma to the Viscosity OF THE BLOOD.}

The viscosity of the blood is directly proportional to that of the plasma, for the same volume of corpuscles, over a very wide range of plasma viscosity (Table VI and Fig. 2).

\section{TABLE VI.}

Variation of the viscosity of suspensions of corpuscles with variation in the viscosity of an artificial plasma. Plasma: $7 \%$ gum acacia in $0.9 \%$ saline. Corpuscles: sheep, washed in saline.

\begin{tabular}{|c|c|c|c|c|c|}
\hline Amount of & & & Vin & ity & \\
\hline $\begin{array}{c}\text { of corpuscles } \\
2 \text { co. } \\
2 " \\
2 " \\
2 "\end{array}$ & $\begin{array}{c}0.9 \% \text { saline } \\
1.5 \mathrm{cc} . \\
1.0 \% " \\
.5 " \\
0 "\end{array}$ & $\begin{array}{l}\text { in saline } \\
0.5 \text { cc. } \\
1.0 " \\
1.5 " \\
2.0 "\end{array}$ & $\begin{array}{c}\text { plasma (p) } \\
1.40 \\
1.77 \\
2.34 \\
3.21\end{array}$ & $\begin{array}{c}\text { blood (b) } \\
3 \cdot 22 \\
4 \cdot 06 \\
5 \cdot 36 \\
7 \cdot 73\end{array}$ & $\begin{array}{l}\bar{p} \\
2 \cdot \\
2 \cdot \\
2 \cdot\end{array}$ \\
\hline
\end{tabular}

Bioah xn 
The table gives the result of increasing the viscosity of the plasma in a suspension of corpuscles in saline by the addition of gum acacia. Sheep's

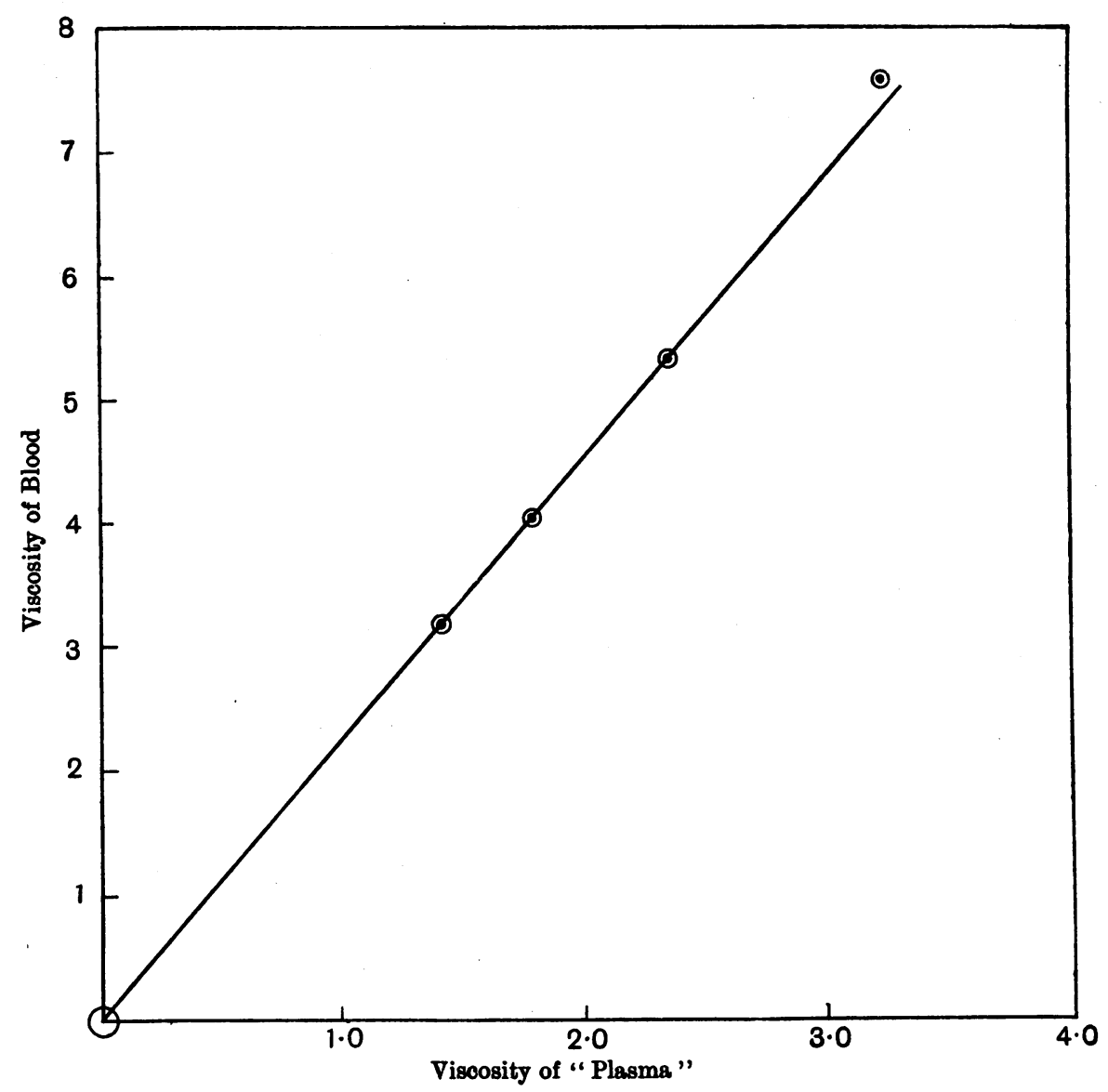

Fig. 2. Graph of relationship of viscosity of blood to that of plasma.

The observed points are seen to lie along a straight line.

corpuscles were washed in $\mathbf{0 . 9} \%$ saline and centrifuged until a fairly concentrated suspension was obtained. This was mixed with varying proportions of $0.9 \%$ saline and $7 \%$ gum acacia in $0.9 \%$ saline as indicated in the table. The viscosity of the various mixtures was determined, as well as that of the "plasma" after the corpuscles had settled. The results are shown and the ratio between "plasma" viscosity and "blood" viscosity given. The constancy of the ratio is obvious while the values of the viscosity plotted against each other lie along a straight line intersecting the abscissa at 0 . The value of the ratio $\frac{b}{p}$ (see Table VI) depends on the volume of the corpuscles present, the influence of which is next considered. 
VI. The Contribution of the Corpuscular Volume to the Viscosity.

The variation in the viscosity of the blood with the volume of the corpuscles present is shown in Table VII. Blood from various sources (indicated) was used, the viscosity of whole blood and plasma and the total volume of the corpuscles present were measured as before described.

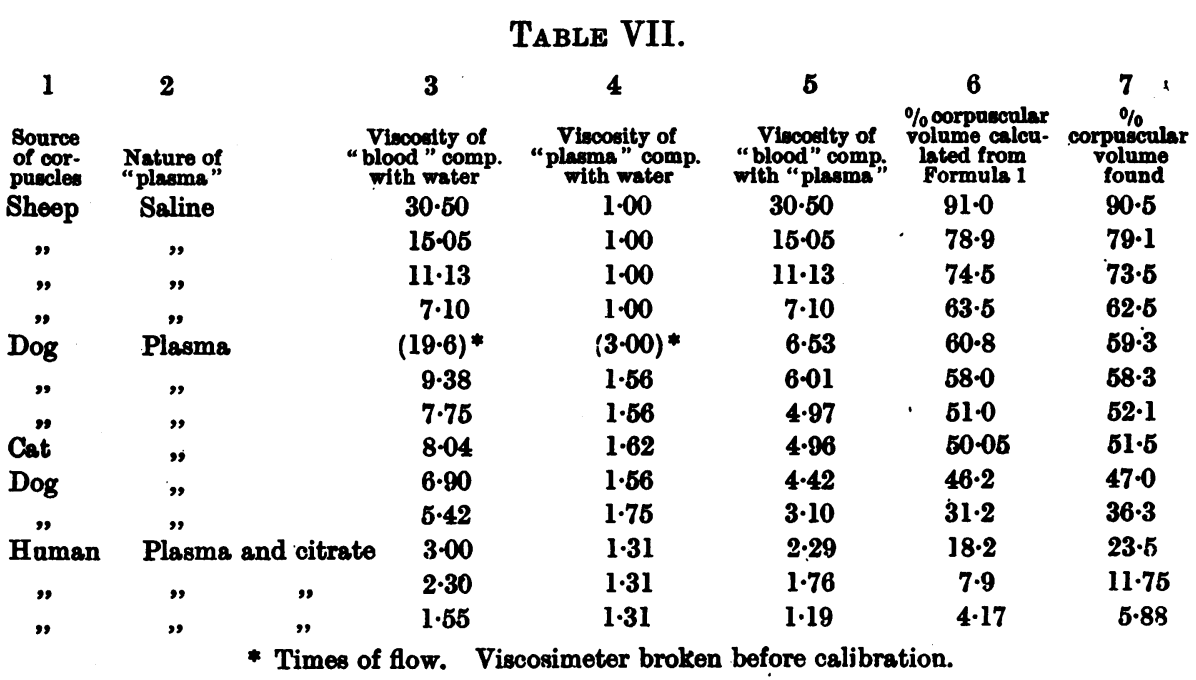

In column 7 of this table are given the haematocrit volumes and in column 6 a series of haematocrit volumes calculated from the formula

$$
{ }^{100}=\left(\frac{n}{n-1}\right)^{3}
$$

where $\boldsymbol{P}=$ volume of corpuscles as $\%$ of total volume of blood.

$n=$ the ratio of the viscosity of the whole blood to that of the plasma.

The agreement above a corpuscular volume of $45 \%$ is within $\pm 1 \%$ which is near the limit of error of the measurements. The curve $A C D$ in Fig. 3 is the curve of the above equation and shows the agreement graphically.

The formula is one established mathematically by Hatschek [1912] for emulsions, when the volume of disperse phase is over $60 \%$ and the rate of shear sufficient to convert the droplets of disperse phase into flat dodecahedra. The viscosity of the disperse phase does not appear in the formula, the only essential being that the disperse phase is easily deformable. This condition is fulfilled by the red corpuscles. The formula does not hold for emulsions below $60 \%$ as at that point the droplets of disperse phase become spherical. In the case of blood corpuscles the discoid shape sufficiently approaches that of a dodecahedron for the formula to hold down to $45 \%$. Below this there 
are other factors, including the rotational energy of the corpuscles, which become increasingly greater as the corpuscles become spaced out, and this, in its turn, causes the calculated and observed values to diverge more and more. The observed values below $40 \%$ lie about a straight line $A B$ which is the graph of

$$
n=1+\frac{K \boldsymbol{P}}{100}
$$

where $n$ and $\boldsymbol{P}$ have the values previously assigned and $\boldsymbol{K}$ is an arbitrary constant $=$ approximately $6 \cdot 3$.

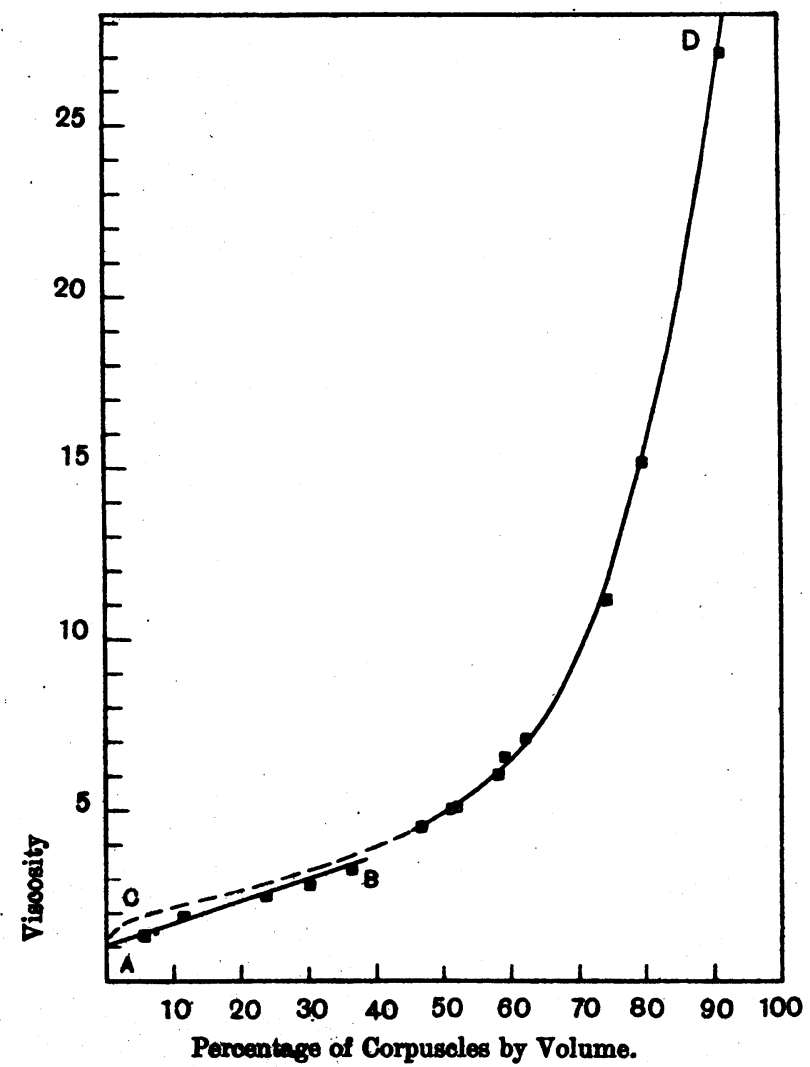

Fig. 3. Belation of viscosity to corpuscular volume.

D obeorved points.

$\angle C D=$ graph of formula 1 .

$A B=$ graph of formula 2.

This formula is similar to that deduced mathematically for suspensions of spheres by Hatschek [1912]

$$
n=1+\frac{4 \cdot 5 P}{100}
$$


but differs in the constant $K$ which, as Hatschek pointed out, depends on the shape of the suspended body. 6.3 was selected as most nearly representing the observed figures. The calculation of the constant for a body of the shape of the corpuscle presents considerable difficulty.

\section{Discussion.}

1. The relationships established above will perhaps be made clear by the following. In narrow passages the blood corpuscles when crowded together move edge foremost. Their distribution next to the wall of the capillary can be represented by the diagram in Fig. 4 . The layer of corpuscles next the wall $(A)$ is practically stationary. That next, at $B$, is travelling slowly, that at $C$ a little faster and so on. The internal friction is produced between the layers of plasma and the surface of the corpuscles: the total kinetic energy of the corpuscles is the same at the beginning and the end of the capillary tube so that the energy degraded into heat in the tube is due entirely to the internal friction of the plasma moving relatively to the corpuscles. This being the case, the conditions can be made clearer by treating the corpuscles as fixed and the plasma as moving along the chinks between them with varying velocities, slowest near the wall and greatest in the centre of the tube. The rate of flow of fluids along such narrow passages was shown by Poiseuille to be inversely proportional to the viscosity. Hence the influence of the plasma on the viscosity of the blood is explained.

He also showed that the rate of flow of fluids along narrow capillary passages is proportional to the fourth power of the radius of the capillary. This explains why the increase of the volume of the corpuscles present, which narrows the passages along which the

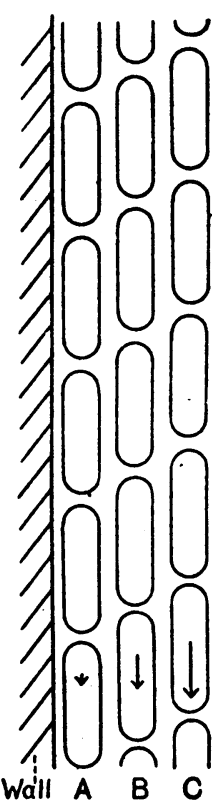

Fig. 4. Diagram of the flow of corpuscles along the wall of the capillary viscosimeter. The arrows represent by their direction and length the direction and velocity of each row of corpuscles. blood flows, produces such a markedly increasing influence on the viscosity of the blood.

2. These experiments suggest a few conjectures as to the shape-consistency of the red corpuscles. Any evidence available goes to show that other living cells are more or less fluid and tend therefore to assume a spherical shape when freed from constraining influences, or unless they are 
definitely amoeboid; e.g. in the numerous cultural experiments with living tissue cells [N. C. Lake, 1916] the growing cells which are found in such cultures are nearly always spherical whatever their origin, unless they become adherent to the lymph or other solid culture medium used. But the red corpuscles, although still undergoing metabolic changes (consumption of $\mathrm{O}_{2}$, glucose [see MacLean 1916] et alia) are flat discs and therefore rigid, if elastic. Even the nucleated corpuscles of birds etc. are flattened. Now consider for a moment what would happen if the red corpuscle were a sphere of liquid protoplasm such as the white corpuscle. If the total volume of corpuscles present were less than $60 \%$ the spheres would be completely separated by plasma, but at $60 \%$ the corpuscular volume would be so great as to cause the spheres to come in contact. Up to that point the viscosity would increase almost in a straight line relationship with the increase in volume, but above it two things may happen depending on the velocity of flow. If the velocity of flow is insufficient to deform the spheres into flat discs the corpuscles would "jam" and cease to flow through the capillaries or only flow exceedingly slowly. Plasma would still flow through the interspaces. Such a condition as this however is much more rarely set up with disc-shaped corpuscles.

3. The white corpuscles have been included in the total volume of corpuscles measured in the above experiments. The rate of shear in the capillary viscosimeters used is probably sufficient to produce in them the requisite change of shape, but in any case their relative volume in most cases is so small as not to affect the results appreciably. In conditions such as myelocytic leukaemia, however, the relative volume of the white corpuscles is so large that considerable modifications of the conditions of capillary flow must arise, and it was hoped, but in vain, that this question of the influence of white cells on the viscosity might have been investigated before publishing these figures. It will at once occur to the reader that the amoeboid properties of the white cell would prevent the block in the capillaries becoming absolute, and that therefore the circulation of dead and of living leucocytes would produce different results.

4. These experiments suggest one or two observations as to the microscopic vascular changes associated with inflammation and infarction. The first stage in the vascular change accompanying inflammation is a dilatation of the capillaries accompanied by an increased rate of flow: this is succeeded by an increased permeability of the capillary wall leading to an increased outpouring of lymph: the rate of flow of the blood then gradually slows down 
and finally almost ceases. It is suggested that this stage of stasis in inflammatory conditions is due not to any special change in the wall but to the very great and increasing rise in the viscosity of the blood consequent on the loss of plasma. This condition of stasis would be commoner than it is were the corpuscles not flattened discs.

\section{SUMmaRY.}

1. A form of capillary viscosimeter for blood is described in which stirring during the observations is possible.

2. Errors and precautions during the measurement are discussed.

3. The viscosity of the blood is shown to be a constant multiple of that of the plasma for the same number of corpuscles.

4. Mathematical relationships are shown to hold between the number of corpuscles and the viscosity.

5. Some of the bearings of the results are discussed.

The work was carried out during the tenure of a grant from the Medical Research Committee to whom my thanks are due.

I have to thank Professor Bainbridge for much kind encouragement and criticism during the progress of the work.

\section{REFERENCES.}

Burton-Opitz (1911), J. Amer. Med. Assoc., 57, 353.

Denning and Watson (1806), Proc. Roy. Soc., B, 78, 328.

Hamburger (1905), Osmotischer Druck und Ionenlehre.

Hatschek (1912), Kolloid, Zeitoch., 11, 280.

Josué and Maturier (1916), Ann. Med., 8, 347.

Lake (1916), J. Physiol., 50, 364.

MacLean (1916), J. Physiol., 50, 168.

McClendon (1916), Physical Chemistry of Vital Phenomena, Princetown Univ. Press.

Reynolds (1883), Phil. Trans., 174, 935. 